3

\title{
Release of Nitrosamines and Nitrosamines Precursors from Scrap Tires
}

$\S$ Department of Environmental Engineering and Earth Sciences, Clemson University, Anderson, SC 29625, United States

${ }^{\dagger}$ Research Center of Environmental Pollution (CICA), University of Costa Rica, 2060 San José, Costa Rica

${ }^{\ddagger}$ Hazen and Sawyer, Fairfax, VA 22030, United States

${ }^{\perp}$ Water Quality Research and Development Division, Southern Nevada Water Authority, Las

Vegas, NV 89193, United States 
Analytical methods

\section{Text S1}

Chemical reagents. Monochloramine stock solutions with a chlorine to nitrogen mass ratio of 4.75:1 were prepared fresh by the slow addition of $\mathrm{NaOCl}$ (Baker Analyzed Reagent, 5\% available free chlorine) to a rapidly stirred $\left(\mathrm{NH}_{4}\right)_{2} \mathrm{SO}_{4}(\mathrm{BDH}, 99 \%)$ solution, the $\mathrm{pH}$ of both solutions was previously adjusted to 9 adding $\mathrm{NaOH}$ and/or $\mathrm{HCl}^{1}{ }^{\text {A }}$ borate buffer $(800 \mathrm{mM}, \mathrm{pH} 8.0)$ was prepared dissolving sodium tetraborate decahydrate (Sigma Aldrich, 99\%) and boric acid (Beantown Chemical, 99\%) in distilled and deionized (DDI) water. Nitrosamines calibration standards were prepared from a nitrosamine mix (EPA 521 Nitrosamine Mix, Supelco). The calibration curves included at least six calibration standards in the range of $0.5-800 \mathrm{ng} / \mathrm{L}$, and the minimum reporting level (MRL) was $0.5 \mathrm{ng} / \mathrm{L}$.

\section{Text S2}

NDMA-d 6 (40 ng/L) (Restek, 99\%) was added as surrogate standard to the quenched samples. Samples were extracted using previously conditioned solid phase extraction cartridges (EU52112M6, UCT) at a flow rate of $10 \mathrm{~mL} / \mathrm{min}$. Then, air was drawn through the cartridge for 10 min at full vacuum. The adsorbed nitrosamines were eluted with methylene chloride and passed through a drying column packed with $6 \mathrm{~g}$ of $\mathrm{Na}_{2} \mathrm{SO}_{4}$ anhydrous for removing residual water. The samples were concentrated to about $0.9 \mathrm{~mL}$ under a gentle stream of ultra-high purity nitrogen gas. $N$-nitroso-di-n-propylamine-d14 $\left(\mathrm{NDPA}_{-} \mathrm{d}_{14}\right)$ was spiked in the samples $(40 \mathrm{ng} / \mathrm{L})$ as an internal standard, and the volume was adjusted to $1 \mathrm{~mL}$ with methylene chloride. Samples were transferred to amber Gas Chromatograph (GC) vials with inserts and $1 \mu \mathrm{L}$ was injected in the front multimode inlet in splitless mode on a Agilent 7890B GC (Santa Clara, CA, US) equipped with 
an Agilent DB-1701 (30 m x $0.25 \mathrm{~mm} \times 1.00 \mu \mathrm{m})$ column, and coupled with an Agilent 7000C triple quadrupole mass spectrometer with electron ionization. ${ }^{2}$

Nitrosamines analysis. Nitrosamines standards were prepared from a nitrosamine mix in

51 methylene chloride (40035-U, Supelco, min. 99.6\%). The calibration curves included at least six

52 standards in the range of $0.5-400 \mathrm{ng} / \mathrm{L}$, and the minimum reporting level (MRL) was $0.5 \mathrm{ng} / \mathrm{L}$.

53 The temperature program for the front multimode inlet was $35^{\circ} \mathrm{C}$ for $0.1 \mathrm{~min}$, then ramped to 265

$54{ }^{\circ} \mathrm{C}$ at a rate of $600{ }^{\circ} \mathrm{C} / \mathrm{min}$. The $\mathrm{GC}$ inlet was held at $280{ }^{\circ} \mathrm{C}$ and lined with an Agilent dimpled

55 ultra-inert liner $\left(2 \mu \mathrm{m}\right.$ id). The oven temperature program was $50{ }^{\circ} \mathrm{C}$ held for 1 min then raised to 56 $80{ }^{\circ} \mathrm{C}$ at a rate of $10{ }^{\circ} \mathrm{C} / \mathrm{min}$, increased to $130{ }^{\circ} \mathrm{C}$ at $20^{\circ} \mathrm{C} / \mathrm{min}$, increased to $230{ }^{\circ} \mathrm{C}$ at a rate of 60

$57{ }^{\circ} \mathrm{C} / \mathrm{min}$ and finally held for $2 \mathrm{~min}$ (total run time $10.17 \mathrm{~min}$ ). The GC-MS/MS transfer line temperature was maintained at $280{ }^{\circ} \mathrm{C}$. The helium carrier gas flow rate was $1.2 \mathrm{~mL} / \mathrm{min}$. 59 Ionization was carried out by electron impact (EI) with an EI voltage of $70 \mathrm{eV}$ and a source 60 temperature of $280{ }^{\circ} \mathrm{C}$. The triple quadrupole $\mathrm{MS}$ detector was operated in multiple reaction 61 monitoring (MRM) mode and two MRM transitions were monitored for NDMA, NDMA-d6 and 62 NDPA-d14 for quantification and confirmation of molecular identification. All samples were run 63 with a solvent delay of $4.3 \mathrm{~min}$. The MRM transitions monitored for all analytes, the specific dwell 64 times and the collision energies are presented in Table S1. The analytical variability across 65 duplicates was $<10 \%$. The quality control consisted of analyzing the recovery of NDMA from 66 two samples prepared in the laboratory with sun exposed distilled and deionized water fortified at $6710 \mathrm{ng} / \mathrm{L}$ and $50 \mathrm{ng} / \mathrm{L}$ and also the analysis of the $10 \mathrm{ng} / \mathrm{L}$ calibration standard measured every 10 68 samples. 
Table S1. Acquisition data for GC-MS/MS analysis of nitrosamines (target), NDMA-d6 (surrogate) and NDPA-d14 (internal standard).

\begin{tabular}{|c|c|c|c|}
\hline Compound & $\begin{array}{l}\text { Retention time } \\
\text { (min) }\end{array}$ & $\begin{array}{c}\text { MRM Transition (Precursor ion } \rightarrow \\
\text { Product ion) }\end{array}$ & $\begin{array}{l}\text { Collision energy } \\
\text { (V) }\end{array}$ \\
\hline \multirow{2}{*}{ NDMA } & \multirow{2}{*}{6.841} & Quantifier: $74.0 \rightarrow 44.0$ & 3.0 \\
\hline & & Qualifier: $74.0 \rightarrow 42.0$ & 7.0 \\
\hline \multirow{2}{*}{ NDMA-d6 } & \multirow{2}{*}{6.957} & Quantifier: $80.0 \rightarrow 50.0$ & 3.0 \\
\hline & & Qualifier: $80.0 \rightarrow 48.0$ & 7.0 \\
\hline \multirow{2}{*}{ NMEA } & \multirow{2}{*}{7.945} & Quantifier: $88.0 \rightarrow 71.0$ & 3.0 \\
\hline & & Qualifier: $88.0 \rightarrow 43.0$ & 5.0 \\
\hline \multirow{2}{*}{ NDEA } & \multirow{2}{*}{8.780} & Quantifier: $102.0 \rightarrow 85.0$ & 5.0 \\
\hline & & Qualifier: $102.0 \rightarrow 56.0$ & 10.0 \\
\hline \multirow{2}{*}{ NDPA-d14 } & \multirow{2}{*}{10.657} & Quantifier: $144.0 \rightarrow 126.0$ & 5.0 \\
\hline & & Qualifier: $144.0 \rightarrow 50.0$ & 5.0 \\
\hline \multirow{2}{*}{ NDPA } & \multirow{2}{*}{10.733} & Quantifier: $130.0 \rightarrow 113.0$ & 5.0 \\
\hline & & Qualifier: $130.0 \rightarrow 43.0$ & 5.0 \\
\hline \multirow{2}{*}{ NMOR } & \multirow{2}{*}{11.137} & Quantifier: $116.0 \rightarrow 56.0$ & 5.0 \\
\hline & & Qualifier: $116.0 \rightarrow 86.0$ & 5.0 \\
\hline \multirow{2}{*}{ NPYR } & \multirow{2}{*}{11.484} & Quantifier: $100.0 \rightarrow 55.0$ & 5.0 \\
\hline & & Qualifier: $100.0 \rightarrow 70.0$ & 5.0 \\
\hline \multirow{2}{*}{ NPIP } & \multirow{2}{*}{11.725} & Quantifier: $114.0 \rightarrow 84.0$ & 5.0 \\
\hline & & Qualifier: $114.0 \rightarrow 97.0$ & 5.0 \\
\hline \multirow{2}{*}{ NDBA } & \multirow{2}{*}{13.279} & Quantifier: $158.0 \rightarrow 99.0$ & 5.0 \\
\hline & & Qualifier: $158.0 \rightarrow 141.0$ & 5.0 \\
\hline
\end{tabular}

3. Text S3

74

Standard Methods (SM) ${ }^{3}$ were followed to measure dissolved organic carbon (DOC) and dissolved nitrogen (DN), using a Shimadzu Total Organic Carbon (TOC)- $\mathrm{V}_{\mathrm{CSH}}$ coupled with the TNM-1

77 (SM $5310 \mathrm{~B}$ ); free chlorine and monochloramine using the $N, N$-diethyl-p-phenylenediamine (DPD) Ferrous Titrimetric Method (SM 4500-Cl F); UV absorbance using a Varian Cary 50 (SM 
5910); $\mathrm{pH}$ using a VWR Symphony $\mathrm{pH}$ meter (SM 4500- $\left.\mathrm{H}^{+}\right)$; and U.S. EPA methods ${ }^{4}$ to measure nitrate and nitrite with a Dionex ICS-2100 Ion Chromatograph (U.S. EPA Method 300). Ammonia concentrations were measured with a $\mathrm{HACH}$ spectrophotometer. Dissolved organic nitrogen

82 (DON) values were calculated by subtracting ammonia, nitrite and nitrate from DN. Minimum reporting levels (MRL) are presented in Table S2.

Table S2. Analytical methods and minimum reporting levels (MRL).

\begin{tabular}{|l|c|c|c|}
\hline \multicolumn{1}{|c|}{ Parameter } & Method & Equipment & $\begin{array}{c}\text { MRL or } \\
\text { Accuracy }\end{array}$ \\
\hline Ammonia (mg/L) & Salicylate & HACH Test Kit & 0.02 \\
\hline DN (mg/L) & $\begin{array}{c}\text { High Temp. } \\
\text { Combustion }\end{array}$ & $\begin{array}{c}\text { Shimadzu TOC-V } \\
\text { TNS \& }\end{array}$ & 0.1 \\
\hline DOC (mg/L) & SM 5310 B & Shimadzu TOC-V CHS & 0.1 \\
\hline Free chlorine (mg/L) & SM 4500-Cl F & N/A & 0.05 \\
\hline Nitrate (mg/L) & EPA 300.0 & Dionex ICS-2100 & 0.005 \\
\hline Nitrite (mg/L) & EPA 300.0 & Dionex ICS-2100 & 0.005 \\
\hline Nitrosamines (ng/L) & EPA 521 & $\begin{array}{c}\text { Agilent 7890B coupled } \\
\text { to 7000C MS/MS }\end{array}$ & 0.5 \\
\hline pH & SM 4500-H ${ }^{+}$ & VWR Symphony & \pm 0.01 \\
\hline Residual combined chlorine $(\mathrm{mg} / \mathrm{L})$ & SM 4500-Cl F & N/A & 0.05 \\
\hline
\end{tabular}

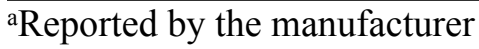

Table S3. Occurrence of nitrosamines (no oxidant) in crumb rubber ( $8 \times 14$ US mesh) and tire chips after leaching test. Experimental conditions: $\mathrm{pH}=7.0$, contact time $=24 \mathrm{~h}$.

\begin{tabular}{|c|c|c|c|}
\hline \multirow{2}{*}{ Nitrosamine } & \multicolumn{3}{|c|}{ Concentration (nmol/g tire) } \\
\cline { 2 - 4 } & Crumb rubber & $2.5 \mathrm{~cm} 2.5 \mathrm{~cm}$ & $10 \mathrm{~cm} \times 5 \mathrm{~cm}$ \\
\hline NMOR & 9.6 & 3.7 & 0.6 \\
\hline NDMA & 1.5 & 0.8 & 0.8 \\
\hline NDPA & 0.2 & $<\mathrm{MRL}$ & $<\mathrm{MRL}$ \\
\hline NPYR & 0.2 & $<\mathrm{MRL}$ & $<\mathrm{MRL}$ \\
\hline NMEA & $<\mathrm{MRL}$ & $<\mathrm{MRL}$ & $<\mathrm{MRL}$ \\
\hline NDEA & $<\mathrm{MRL}$ & $<\mathrm{MRL}$ & $<\mathrm{MRL}$ \\
\hline NPIP & $<\mathrm{MRL}$ & $<\mathrm{MRL}$ & $<\mathrm{MRL}$ \\
\hline NDBA & $<\mathrm{MRL}$ & $<\mathrm{MRL}$ & $<\mathrm{MRL}$ \\
\hline
\end{tabular}


Table S4. Nitrosamine analysis of crumb rubber ( 8 x 14 US mesh) and tire chips after ozonation.

\begin{tabular}{|l|c|c|c|}
\hline \multirow{2}{*}{ Nitrosamine } & \multicolumn{3}{|c|}{ Concentration (nmol/g tire) } \\
\cline { 2 - 4 } & Crumb rubber & $2.5 \mathrm{~cm} \times 2.5 \mathrm{~cm}$ & $10 \mathrm{~cm} \times 5 \mathrm{~cm}$ \\
\hline NMOR & 10.5 & 3.1 & 0.5 \\
\hline NDMA & 1.6 & 0.8 & 0.5 \\
\hline NDPA & 0.2 & $<\mathrm{MRL}$ & $<\mathrm{MRL}$ \\
\hline NMEA & $<\mathrm{MRL}$ & $<\mathrm{MRL}$ & $<\mathrm{MRL}$ \\
\hline NPYR & $<\mathrm{MRL}$ & $<\mathrm{MRL}$ & $<\mathrm{MRL}$ \\
\hline NDEA & $<\mathrm{MRL}$ & $<\mathrm{MRL}$ & $<\mathrm{MRL}$ \\
\hline NPIP & $<\mathrm{MRL}$ & $<\mathrm{MRL}$ & $<\mathrm{MRL}$ \\
\hline NDBA & $<\mathrm{MRL}$ & $<\mathrm{MRL}$ & $<\mathrm{MRL}$ \\
\hline
\end{tabular}

92

93

94

95

\begin{tabular}{|c|c|c|c|c|c|c|}
\hline \multirow[t]{2}{*}{ Parameter } & \multicolumn{2}{|c|}{ pH 4} & \multicolumn{2}{|c|}{ pH 7.0} & \multicolumn{2}{|c|}{ pH 10.0} \\
\hline & $12 \mathrm{~h}$ & $24 \mathrm{~h}$ & $12 \mathrm{~h}$ & $24 \mathrm{~h}$ & $12 \mathrm{~h}$ & $24 \mathrm{~h}$ \\
\hline $\mathrm{DOC}(\mathrm{mg} / \mathrm{L}$ as $\mathrm{C})$ & 14.4 & 18.1 & 14.1 & 16.7 & 13.9 & 17.0 \\
\hline $\mathrm{DN}(\mathrm{mg} / \mathrm{L}$ as $\mathrm{N})$ & 1.1 & 1.4 & 1.1 & 1.3 & 1.1 & 1.3 \\
\hline $\mathrm{UV}_{254}\left(\mathrm{~cm}^{-1}\right)$ & 0.1880 & 0.2311 & 0.1896 & 0.2306 & 0.2278 & 0.2743 \\
\hline $\operatorname{SUVA}_{254}(\mathrm{~L} / \mathrm{mg} \cdot \mathrm{cm})$ & 1.3 & 1.3 & 1.3 & 1.4 & 1.6 & 1.6 \\
\hline
\end{tabular}

Table S5. Effect of $\mathrm{pH}$ and contact time on the release of DOC tire crumb rubber $(8 \times 14$ US mesh).

6 


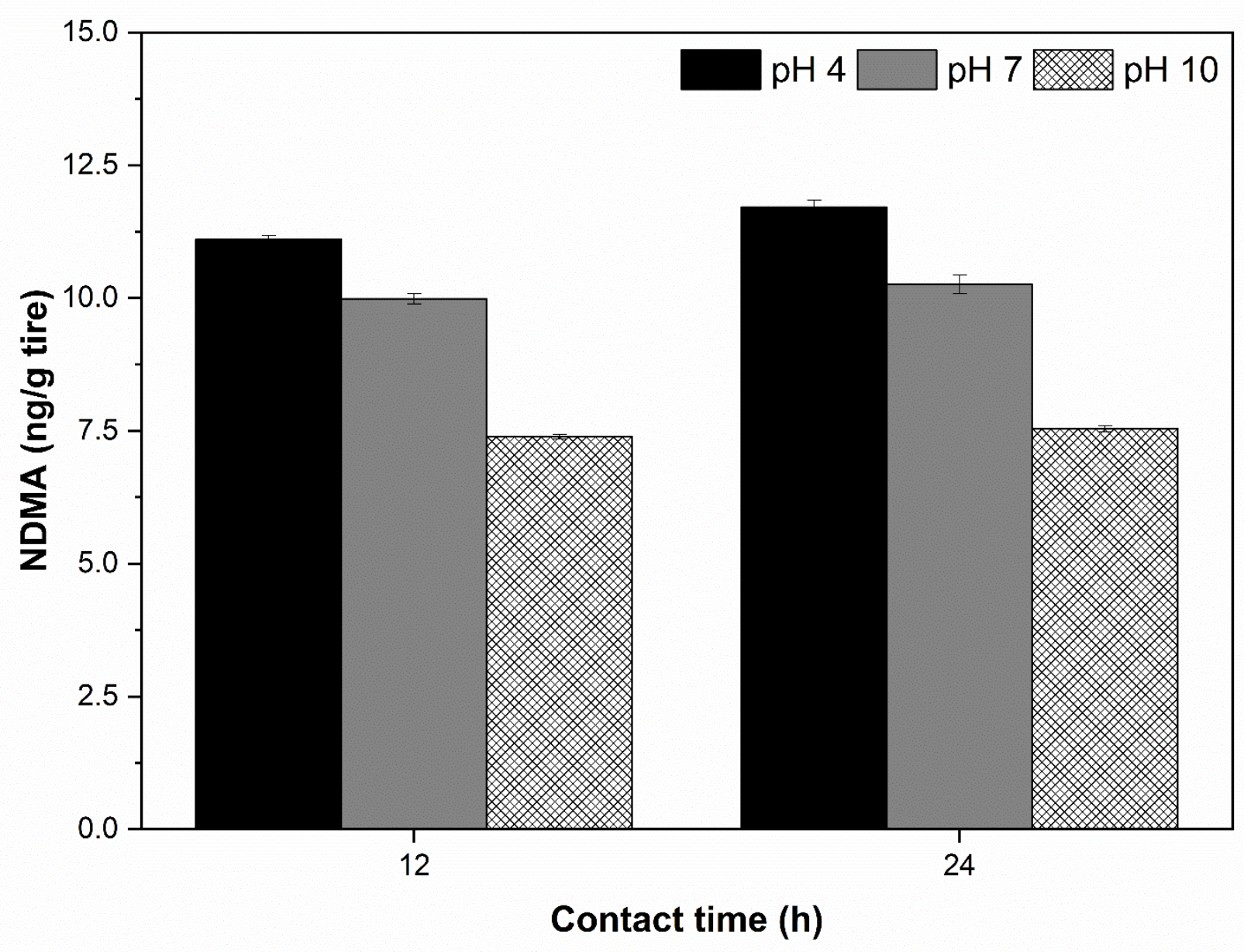

97

Figure S1. Effect of $\mathrm{pH}$ and contact time on the release of NDMA precursors from crumb rubber 99 (8 x 14 US mesh).

100 


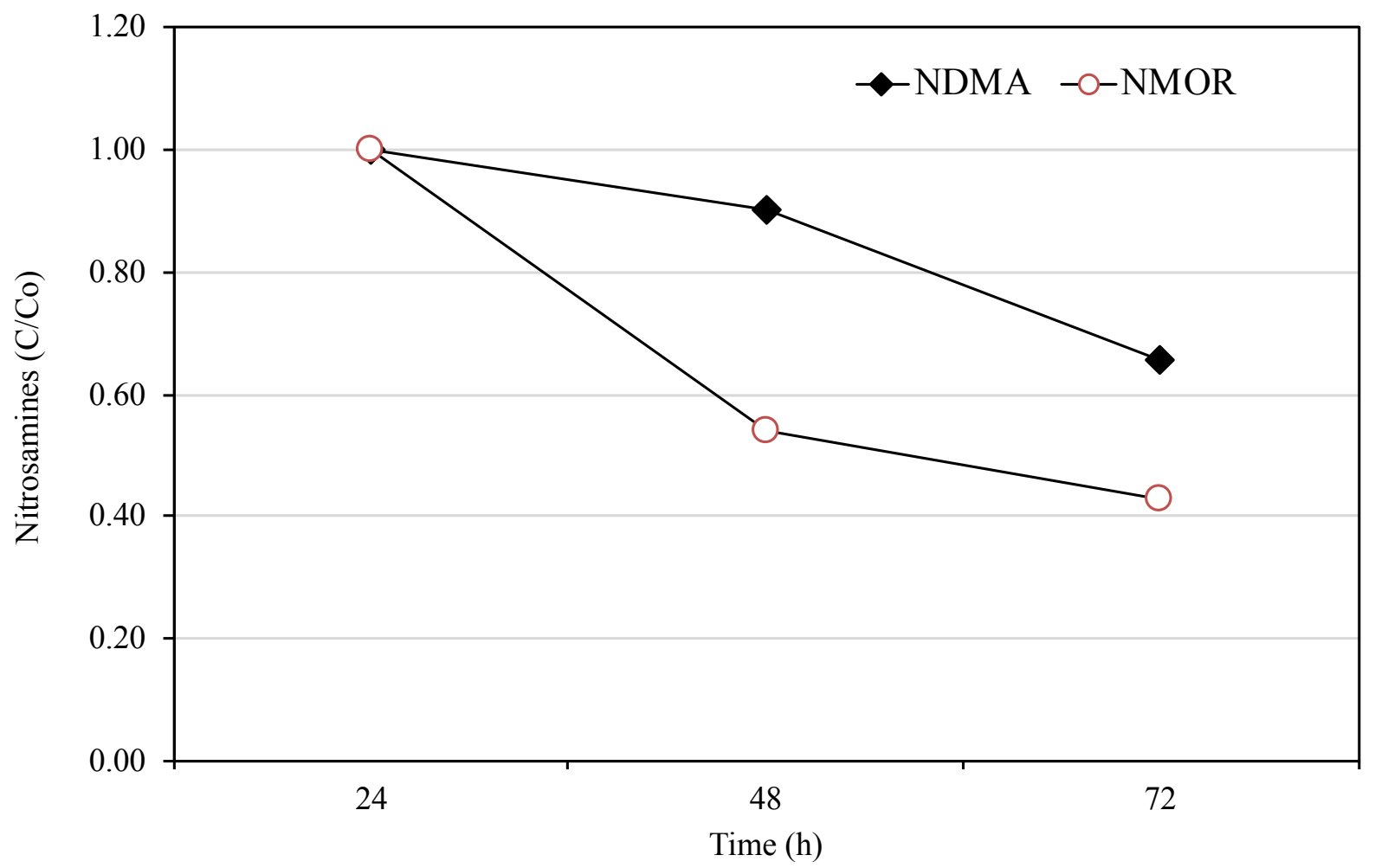

101

102 Figure S2. Effect of sequential cycles of leaching of scrap times on the release of NDMA and 103 NMOR precursors. Experimental conditions: $\mathrm{pH}=7.0,\left[\mathrm{NH}_{2} \mathrm{Cl}\right]=100 \mathrm{mg} / \mathrm{L}$ as $\mathrm{Cl}_{2}$.

References:

106

(1) Hong, Y.; Liu, S.; Song, H.; Karanfil, T. HAA Formation during Chloramination-Significance of

107

108

109

110

111

112

113

114

115

116 by Isotope Dilution Gas Chromatography-Electron Ionisation Tandem Mass Spectrometry. Talanta 2012, 99, 146-154.

(3) APHA; AWWA; WEF. Standard Methods for the Examination of Water and Wastewater, 20th ed.; American Public Health Association: Washington, DC, USA, 2005.

(4) US EPA. Integrated Risk Information System Home Page. Office of Research and Development (ORD), National Center for Environmental Assessment http://www.epa.gov/iris/subst/0045.htm (accessed Nov 11, 2017). 